\title{
RF COUPLING INTO THE FUEL TANK OF A LARGE TRANSPORT AIRCRAFT FROM INTENTIONALLY TRANSMITTING PEDS IN THE PASSENGER CABIN
}

\author{
Truong X. Nguyen, Kenneth L. Dudley, Stephen A. Scearce and Jay J. Ely \\ NASA Langley Research Center, Hampton, VA
}

Robert E. Richardson and Michael O. Hatfield

Naval Surface Warfare Center, Dahlgren, VA

\begin{abstract}
An investigation was performed to study the potential for radio frequency $(\mathrm{RF})$ power radiated from Portable Electronic Devices (PEDs) to create an arcing/sparking event within the fuel tank of a large transport aircraft. This paper describes the experimental methods used for measuring RF coupling to the fuel tank and Fuel Quantity Indication System (FQIS) wiring from PED sources located in the passenger cabin. To allow comparison of voltage/current data obtained in a laboratory chamber FQIS installation to an actual aircraft FQIS installation, aircraft fuel tank RF reverberation characteristics were also measured. Results from the measurements, along with a survey of threats from typical intentional transmitting PEDs are presented. The resulting worst-case power coupled onto fuel tank FQIS wiring is derived. The same approach can be applied to measure RF coupling into various other aircraft systems.
\end{abstract}

\section{Introduction}

Separate papers $[1,2]$ describe the approach and the results from an investigation into RF threats to the Fuel Quantity Indication System (FQIS) wiring. This work was in support of the investigation of the TWA-800 accident in 1996 at the request of the National Transportation Safety Board. The goal was to determine whether there was enough RF energy/power on the FQIS wiring to create a sparking/arcing event inside the aircraft's Center Wing Tank (CWT). A sparking/ arcing event in the CWT may increase the probability of creating an explosion in the tank, leading to a catastrophic accident.

RF threats include sources external and internal to an aircraft. External RF threats, as provided by the Joint Spectrum Center [3], include the worst case RF environment at the aircraft at the time of the accident. They were calculated based on known data about the transmitters and the distance to the aircraft.

RF sources internal to the aircraft include portable electronics devices (PEDs), which consist of intentionally transmitting devices such as portable phones and two-way radios, and unintentionally transmitting devices such as laptop computers and game devices. In terms of total radiated power, intentionally transmitting PEDs are much more likely to be a threat as many of them may radiate up to 6 watts of RF power.

Unintentionally transmitting devices radiate at levels below intentional radiators, even when damaged, and are not considered as a threat in this case.

This paper focuses on the measurement of the potential threats to the CWT and its wiring due to intentionally transmitting PEDs in the passenger cabin. The approach includes identifying PED threats and performing measurements on a retired Boeing 747 airplane of the RF coupling to the CWT and its wiring. Together, the PED threats and the worst case coupling factors provide an upper bound on the maximum threats to the fuel tank and it's wiring. This result is then compared with the power requirement for creating a spark/arc, as determined through laboratory testing, to determine if there 
exists a possibility of RF induced spark/arc in the fuel tank.

Also described in this paper are the measurements of the fuel tank's reverberation characteristics and the RF coupling between different compartments in the fuel tank. These measurements helped in simulating the RF environment in the tank for laboratory FQIS wiring susceptibility testing.

\section{Estimating Typical PED Threats}

PED threats considered in this effort were limited to intentionally transmitting and commercially available devices. Devices developed for military use and those modified to exceed the regulation limits were excluded. Unintentional transmitters, such as computer laptops, were not considered due to their very low radiated power.

In this effort, the PED threats information was gathered from three sources: devices' manufacturer specifications, ANSI C63.18-1997 for typical transmitters, and FCC limits. A short description of each source is described below.

\section{Manufacturer Specifications}

Specifications on frequencies and the maximum radiated power were gathered for about 50 devices. These devices included portable radios, cellular and PCS phones, satellite phones, wireless LAN, and two-way pagers [1]. A summary plot of the data is shown in Figure 1.

\section{ANSI C63.18-1997}

ANSI C63.18-1997 standard [4] describes a recommended practice for estimating immunity of medical devices to specific RF transmitters. In addition, it provides a table of typical PEDs and their maximum output power levels. Some data from the standard that are useful to this effort are shown in Table 1. Other PEDs data from the standard are omitted from Table 1 due to their very low probability of being used on an airplane. Examples include $25 \mathrm{~W}$ mobile radios and 10-100 $\mathrm{W}$ police/ambulance radios, which are typically mounted to a vehicle. Also omitted are CISPR 11 and CISPR 22 devices due to extremely low radiated power levels that are in fractions of a microwatt. Data from Table 1 are also plotted in Figure 1.

Table 1. PEDs and maximum output power

\begin{tabular}{|l|c|c|}
\hline \multicolumn{1}{|c|}{ Product } & $\begin{array}{c}\text { Frequency } \\
\text { (MHz) }\end{array}$ & $\begin{array}{c}\text { Power } \\
\text { (W) }\end{array}$ \\
\hline Hand-held transceivers & $27,49,138-470$ & 5 \\
\hline Wireless LANs & 912,2400 & 0.1 \\
\hline Personal digital assistants & $896-940$ & 4 \\
\hline Radio modems & $896-901$ & 10 \\
\hline Cellular Telephones & $800-900$ & 0.6 \\
\hline Personal satellite telephones & $1610-1626.5$ & 1 \\
\hline Licensed PCS equipment & $1850-1910$ & 1 \\
\hline
\end{tabular}

\section{Determination of FCC limits}

With the exception of the federal government spectrum, the maximum radiated power from any legally used PED in the U.S. must be below the FCC limits, by regulation. Thus, FCC limits were of interest since they provided an upper bound for all commercially available devices, except those using federal government frequencies. However, a manual search through the FCC regulations proved tedious. Many specifications were ambiguous as the power levels varied depending on uses, even though they shared the same spectrum. Many specifications were vague or were not yet defined. As a result, the gathered data on the FCC limits were incomplete, but useful for this level of analysis. The compiled FCC limits data are also shown in Figure 1.

\section{Summary of PED Threat Levels}

As can be seen from Figure 1, most devices radiate six watts or less, except at $27 \mathrm{MHz}$ and at $900 \mathrm{MHz}$. The FCC limit at $27 \mathrm{MHz}$ is $25 \mathrm{~W}$ for remote radio control, and the ANSI standard shows $10 \mathrm{~W}$ maximum for radio modem at $900 \mathrm{MHz}$. Figure 1 also shows that PEDs frequencies were mostly limited to between $25 \mathrm{MHz}$ and $2.6 \mathrm{GHz}$. The FCC allocates spectrum to about $6 \mathrm{GHz}$ for a few applications, however. Based on this data, the frequency range for the coupling measurement at the aircraft was chosen to be from $25 \mathrm{MHz}$ to 6 $\mathrm{GHz}$. Details about this coupling measurement are described in the following sections. 


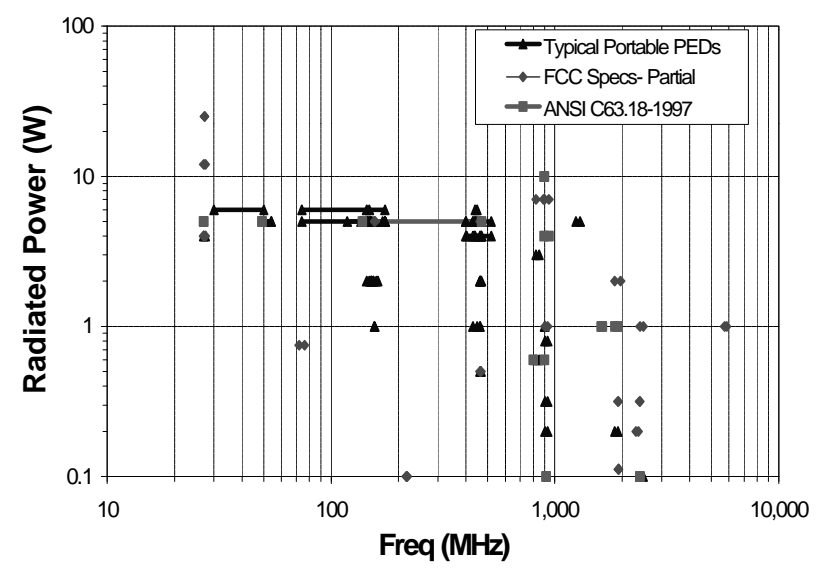

Figure 1. PED threat levels.

\section{Measurements of Coupling to Fuel Tank and Wiring}

There were three assumed paths for coupling RF energy to a fault location in the fuel tank. These paths can be grouped into two coupling groups:

- Conducted Coupling:

Once coupled onto FQIS wiring, RF power stayed attached to wiring all the way to the fault location to create a spark/arc.

- $\quad$ Radiated Coupling:

○ Through FQIS Wiring: RF power coupled into the tank through FQIS wiring then radiated. Energy then re-couples back onto the FQIS at or near the fault location to create a spark/arc.

- Not through FQIS Wiring: RF radiated fields coupled into the CWT through other means such as pipes and unshielded apertures. Energy then re-couples on to the FQIS wiring near a fault location to create a spark/arc.

It was important to measure and bound the power coupled into the CWT and its wiring. A wide-band log-periodic antenna and a dual ridge horn antenna were used in the frequency range from $25 \mathrm{MHz}$ to $6 \mathrm{GHz}$ to simulate radiating PED sources. A special procedure was used to account for antenna mismatch, allowing for out-of-band use of the log periodic antenna between $25 \mathrm{MHz}$ to 100 $\mathrm{MHz}$. By measuring the induced power on the FQIS wiring at the fuel tank connector and the radiated power density in the fuel tank, the coupling data and the bounds were estimated.

\section{Conducted Coupling to CWT on FQIS wiring}

The FQIS wiring provided a conducted coupling path into the CWT. This section describes the coupling measurement from the passenger cabin onto the CWT FQIS wiring. The measurement was performed at the FQIS CWT connector, with an antenna in the passenger cabin simulating the PED sources.

\section{Test Method:}

A diagram of the set up is shown in Figure 2. Power coupled on the FQIS was measured at the fuel tank connector using a spectrum analyzer and a custom adapter. Due to limited time available at the airplane, it was decided to measure power across only three pin combinations that represent both differential and common mode couplings: $\mathrm{HI} Z$ to LO Z pins, HI Z to LO Z COMP, and all pins (HI Z, LO $Z$ and LO $Z$ COMP) tied together relative to airframe chassis. A diagram of the FQIS connector is shown in Figure 3.

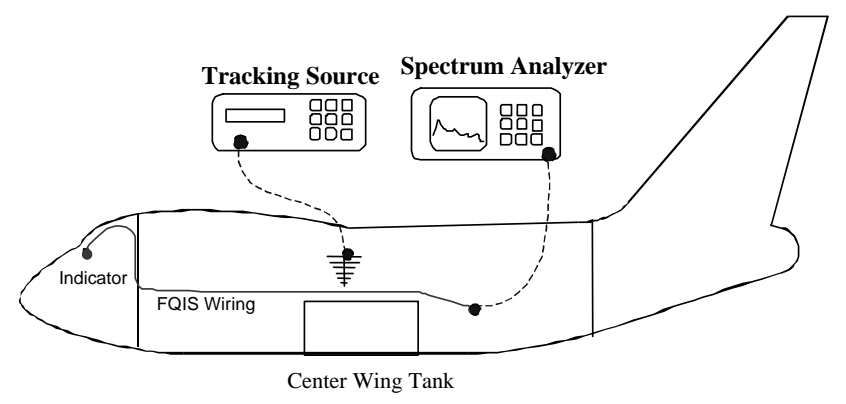

Where:

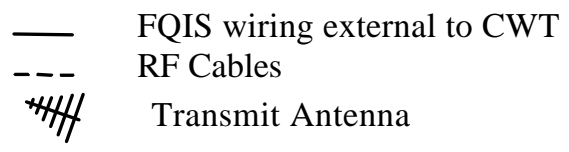

Figure 2. Coupling to FQIS measurement.

The transmit antennas in the passenger cabin were pointed through the floor directly at the FQIS wiring while being moved along the length of the wiring. The spacing between the antenna and wiring was maintained between $5-10 \mathrm{~cm}$. Measurements were performed with antenna polarization both parallel and perpendicular to the wires. A fixed amount of RF power was delivered to the transmit antenna, while the frequency was swept slowly over the measurement band repeatedly. A spectrum 
analyzer, set on maximum trace hold mode, was used to measure the peak coupled power onto the FQIS.

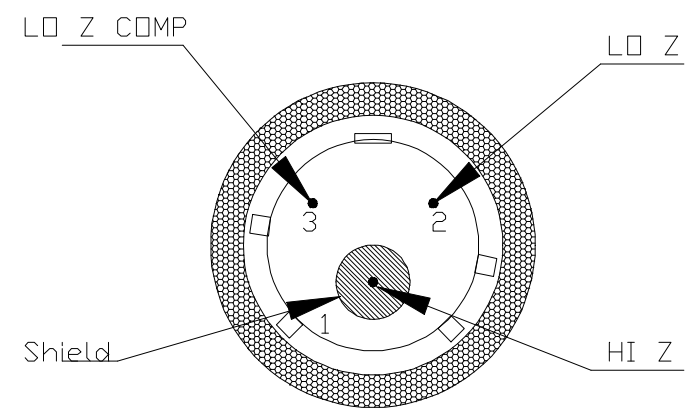

Figure 3. FQIS CWT Amphenol D3 connector schematic.

Figure 4 shows the measurement being performed in the passenger cabin for frequencies below $1 \mathrm{GHz}$. In this image, the transmit antenna was pointing at the FQIS wiring, which was directly under the floor running along the body of the aircraft. The image shows the antenna polarization perpendicular to the wiring. Measurements with antenna polarization parallel to the wiring were also performed.

In addition to pointing directly at the wiring, the transmit antennas were also pointed in several random directions to check for possible coupling paths other than through the FQIS wiring. However, random directions show much lower coupling than by directly illuminating the FQIS wiring.

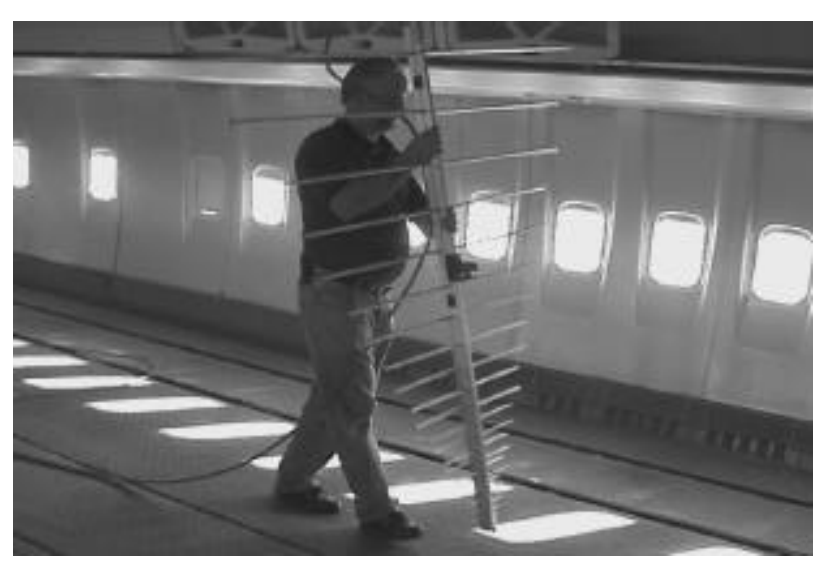

Figure 4. A log-periodic antenna simulating a portable transmitter in the passenger cabin.

\section{Test Results:}

Figure 5 shows the results from the aircraft measurement. Data are presented in two plots due to two separate hardware set-ups necessary to cover the frequency range of interest.

Of the three measurements, the All-Pins to Chassis measurement shows the highest coupling up to about $2 \mathrm{GHz}$. This data represents the common mode coupling, versus differential mode coupling for the other two measurements. Above 2 $\mathrm{GHz}$, all three measurements very much show the same maximum coupling envelope.

It is important to note that the transmit antenna was used out of band below $100 \mathrm{MHz}$. This resulted in high reflected power due to antenna mismatch. To simulate full radiated power, the results were numerically scaled using the scale factor computed from free space antenna reflection coefficients. This approach was used in the past with good results [5].
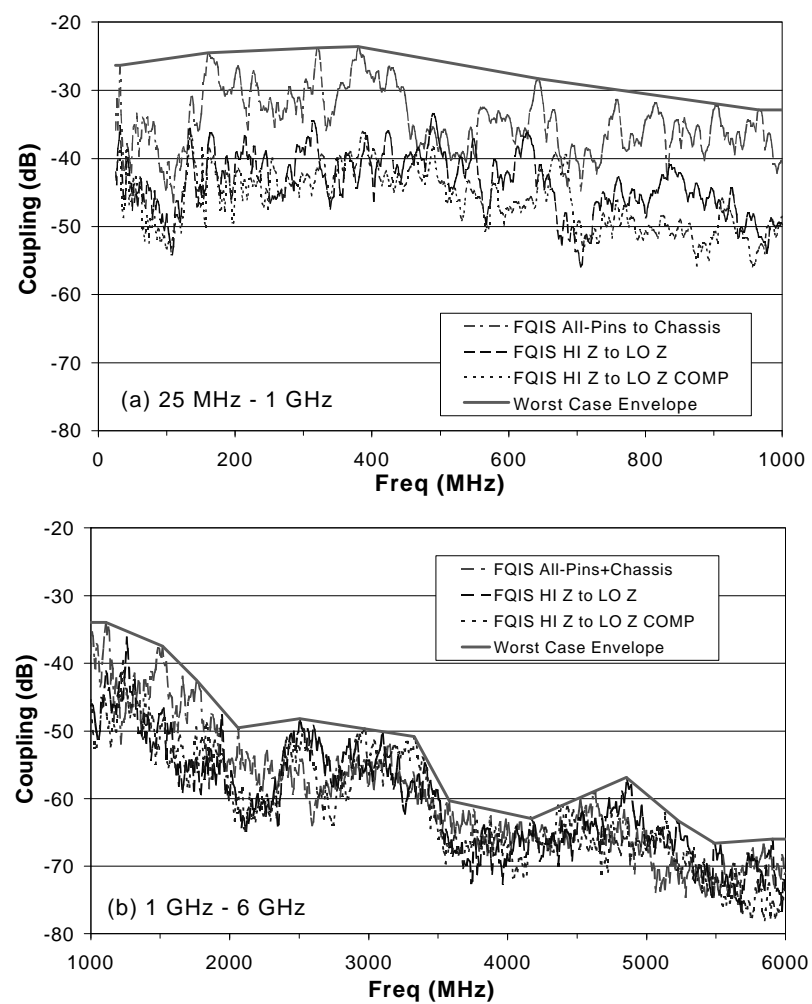

Figure 5. Coupling from passenger cabin to FQIS wiring.

A variation of this correction was also applied to correct for the impedance mismatch caused by the custom adapter. This custom adapter allowed measurement of power on the FQIS wiring using a 
50 ohms $\mathrm{N}$ connector. Without this correction, the mismatch errors can be as much as $4 \mathrm{~dB}$ at $25 \mathrm{MHz}$.

\section{Radiated Coupling to Antenna inside CWT}

Once coupled onto the FQIS wiring, the RF power may propagate on the wiring to the fault location in the CWT, or if the wiring is long, much of the energy may be radiated. Some of the radiated energy may be coupled back onto the FQIS near a fault location to create a spark if the level is high enough. This section describes the measurement of radiated power in the CWT due to PED sources in the passenger cabin, with the FQIS connector to the tank mated as well as de-mated. The results provided an estimate of the upper bounds to the radiated coupling to the inside of the tank, and the amount of coupling through the FQIS wiring.

\section{Test Method:}

The measurement approach was similar to that described previously in measuring the coupling onto FQIS wiring, except that antennas were used to measure power density in the CWT instead. A mechanical stirrer was used to stir RF energy in each of the bays where the measurements were made. This ensured that the receive antenna detected peak power density in that bay. The set up is illustrated in Figure 6.

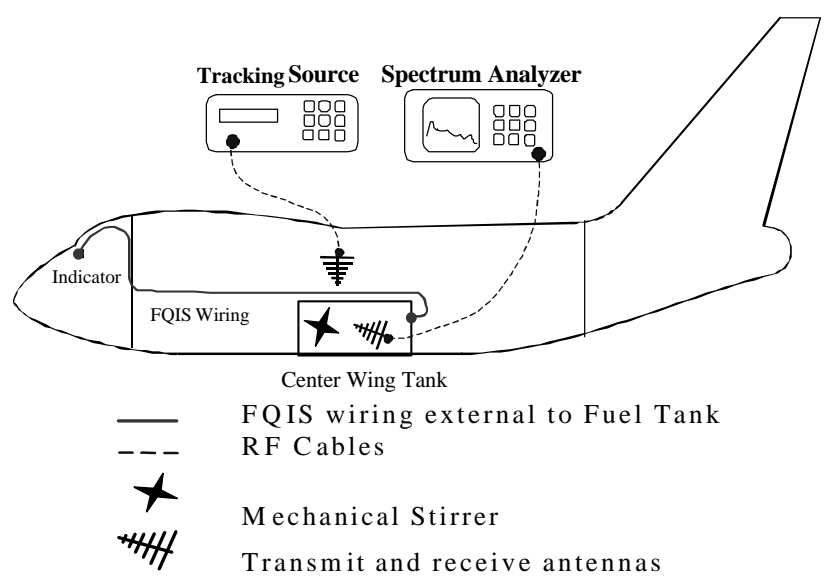

Figure 6. Radiated field coupling to CWT

Due to limited time available at the airplane, radiated field measurements were made in only three of the six bays, covering the bay where the FQIS entered the CWT, the smallest bay, and the largest bay. These correspond to bay 1 , bay 3 , and bay 6 in Figure 7, respectively. The same measurements were repeated with the FQIS connector to the tank de-mated to study the contribution of the FQIS wiring on the CWT radiated field coupling.

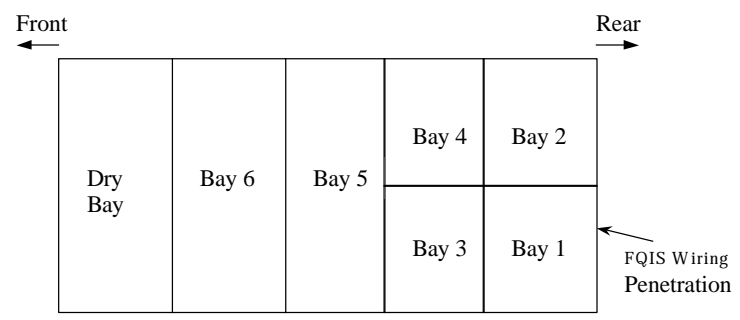

Figure 7. CWT top view. Data was collected for Bay 1 , Bay 3 and Bay 6.

It is important to note that the coupling measurement should provide an upper bound to the power that can be coupled onto the FQIS wiring, since the antennas used in the CWT were expected to be more efficient in coupling RF energy than the FQIS wiring.

Measured data were confirmed to be within the spectrum analyzer's dynamic range. This was important especially with the FQIS connector demated since the coupling values were very low. A pre-amplifier was necessary above $1 \mathrm{GHz}$ for the required instrument sensitivity.

The log periodic antenna in the CWT was used out of band below $200 \mathrm{MHz}$. Unlike the transmit antenna in the passenger cabin, no correction factors were applied to the result to correct for antenna mismatch. The antennas were situated in tight cavity spaces, and these cavity effects on antenna mismatch were difficult to quantify. It was decided best to present the data without the correction.

\section{Test Results:}

With FQIS Connector Mated to the Tank:

The results are shown in Figure 8. The figure shows coupling to bay 1 (where the FQIS entered the CWT) dominates between $500 \mathrm{MHz}$ to $2 \mathrm{GHz}$, while coupling to bays 3 and 6 dominated above 3 GHz. The worst-case coupling was at about 500 $\mathrm{MHz}$, with approximately $-43 \mathrm{~dB}$ coupling factor. This was about $20 \mathrm{~dB}$ lower than the worst case coupling onto the FQIS wires.

With FQIS Connector De-Mated:

For frequencies below $1.5 \mathrm{GHz}$, the peak coupling into the CWT was lowered by about $10 \mathrm{~dB}$ 
on the average when the FQIS connector was demated. This result shows that the FQIS wiring was the major contributor to the radiated field environment in the CWT in this frequency range. Above $1.5 \mathrm{GHz}$, there were no significant differences in the envelopes, whether the connector was mated or de-mated. Further information on this result is presented in Reference [1].
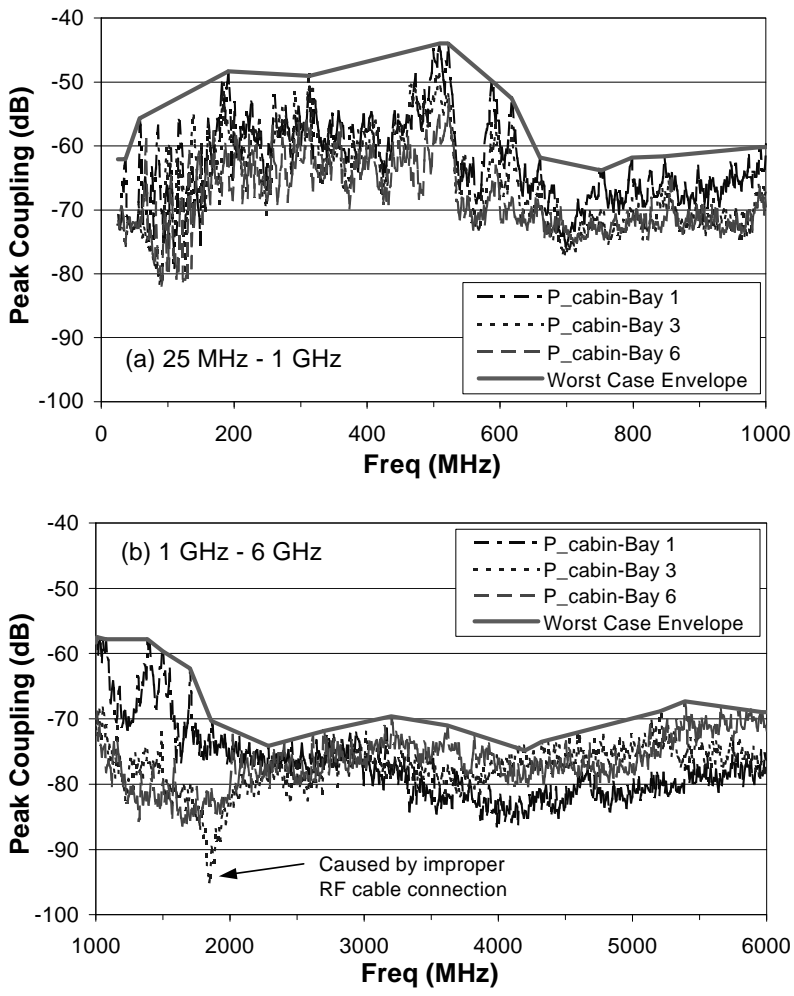

Figure 8. Peak coupling from passenger cabin to antenna in different bays in the CWT. With FQIS connector mated to CWT.

\section{Maximum PEDs power on FQIS wiring and antenna in CWT}

Application of the peak coupling data envelopes in Figures 5 and 8 to the PED threat levels in Figure 1 resulted in Figures 9 and 10. Figure 9 shows the maximum PEDs power coupled onto the FQIS wiring, measured at the tank entry point. Figure 10 shows the maximum power coupled into an antenna in the fuel tank, as measured in bays 1, 3 and 6 .

Figures 9 and 10 compare the power measured at the FQIS wiring connector to the power received by an antenna within the CWT. At least $20 \mathrm{~dB}$ more power was measured at the FQIS wiring connector than could be coupled onto the receive antenna in the CWT. The power level coupled into an efficient antenna provided an upper bound to the power coupled onto the FQIS wiring through the radiated coupling path.

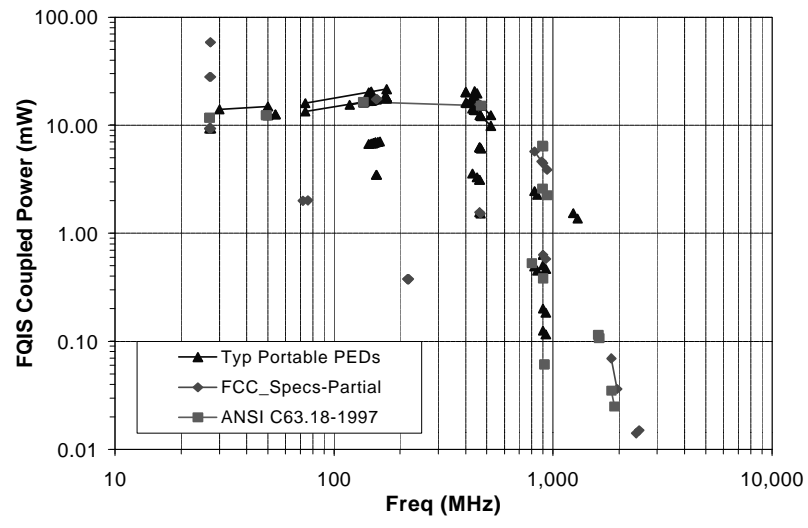

Figure 9. PEDs power coupled onto FQIS wiring.

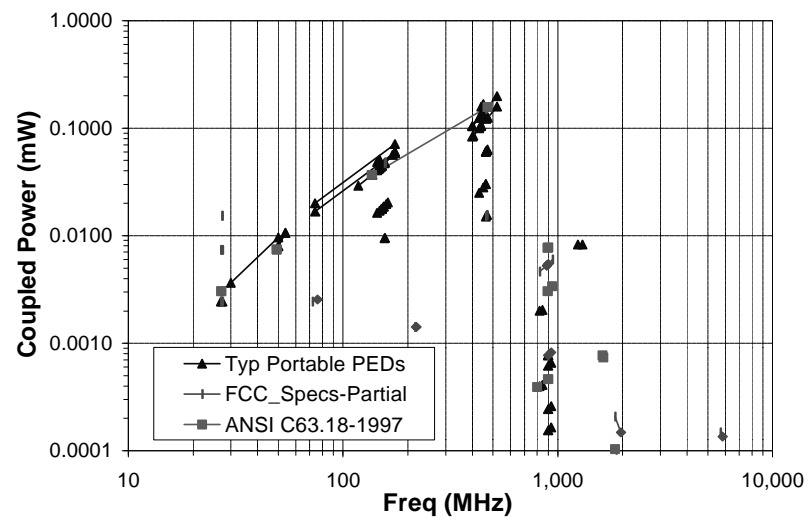

Figure 10. PEDs power coupled into an antenna in CWT.

With the FQIS being the dominant contributor to the radiated field environment in the CWT, and with the CWT's peak cavity coupling factor in the 3-7 dB range (data shown in Figure 12), it can be shown for frequencies between $200 \mathrm{MHz}-1 \mathrm{GHz}$ that only a small fraction of the power on FQIS was radiated in CWT. The majority of the remaining power was lost in the wires, reflected, or lost through other means.

It was also evident that PEDs coupling at frequencies above $1 \mathrm{GHz}$ declines significantly. This result was important for determining the upper frequency limits for subsequent laboratory testing. 


\section{Simulation of CWT Radiated Field Environment}

In order to test for FQIS wiring susceptibility to RF power, conducted and radiated field tests were performed. In the conducted susceptibility test, the desired power was injected onto the FQIS wires. The simulation of the radiated field environment was more involved. The reverberation characteristics of the CWT needed to be characterized and compared to the same parameters measured in a test chamber. The ratio of the CWT data and the test chamber data provided the scale factor necessary in determining the power input into the test chamber.

\section{Cavity Reverberation Measurements}

Procedures for measuring the reverberation parameters are described in Reference [6]. The measurement set-up is illustrated in Figure 11. This procedure involves measuring either the peak or the average power coupling between two antennas in the cavity. Rather than moving the receive antenna to many different locations to sample the power density, it is much more efficient to utilize a mechanical "stirrer" to change cavity field structures as it rotates, while keeping the receive antenna at just one location. A spectrum analyzer is used to measure power coupled into the receive antenna, from which relevant statistics such as the peak and the average values can be extracted.

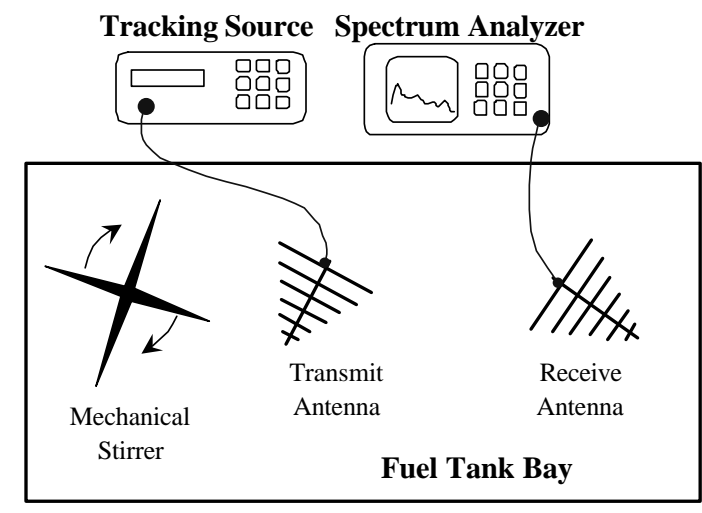

Figure 11. Mode-stirred measurement in a CWT bay.

In simulating the environment of the CWT using a test chamber, it was decided to compare the peak coupling values, instead of averages. Peak cavity coupling resemble the worst case coupling onto the FQIS wiring in the CWT. Peak coupling data was also easier and faster to collect on the aircraft compared to average coupling data, with minimal additional uncertainties for our purposes.

Peak cavity coupling was performed in bay 1 , bay 3, and bay 6 between $25 \mathrm{MHz}$ to $6 \mathrm{GHz}$. The results are shown in Figure 12.
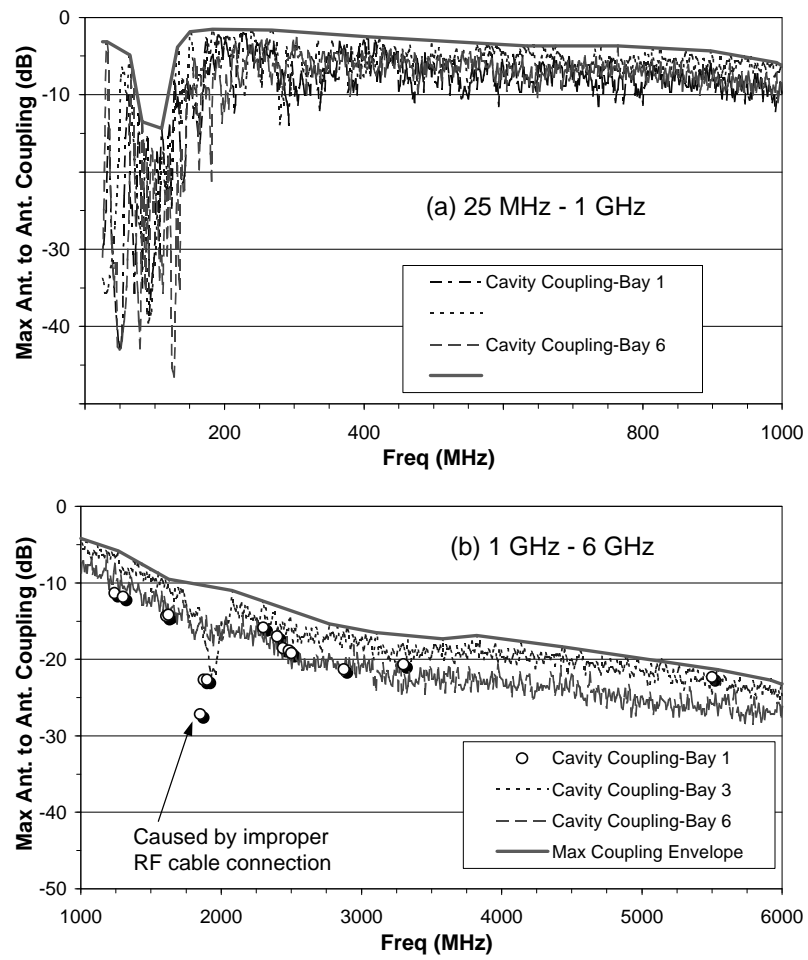

Figure 12. Peak cavity coupling for bays 1, 3 and 6.

The antennas were used out of band below 200 MHz. Thus, data below $200 \mathrm{MHz}$ are shown for completeness only. In addition, bay 1 data was measured at only a few selected PED frequencies. The measurement approach was later changed from discrete frequency stepping to continuous frequency sweeping, allowing for finer frequency increment as shown in other curves in the same plot.

\section{CWT versus Reverberation Chamber Comparison}

Reverberation Test Chamber "B" at NASA Langley Research Center (LaRC) was used to simulate the reverberating environment in the CWT. Johnson [8] shows that RF coupling to 
avionics systems measured in a reverberation chamber is a valid description of the RF coupling characteristics of these same systems over the same frequency range when the systems are installed in aircraft cavities. Cavities in Reference [8] included the passenger cabin, the cockpit, and the avionics bay of a large transport airplane such as a Boeing 707. The CWT had even better reverberation characteristics than those cavities due to having much lower losses associated with having completely closed boundaries. Thus using a reverberation chamber was valid for simulating the reverberation characteristics of the CWT.
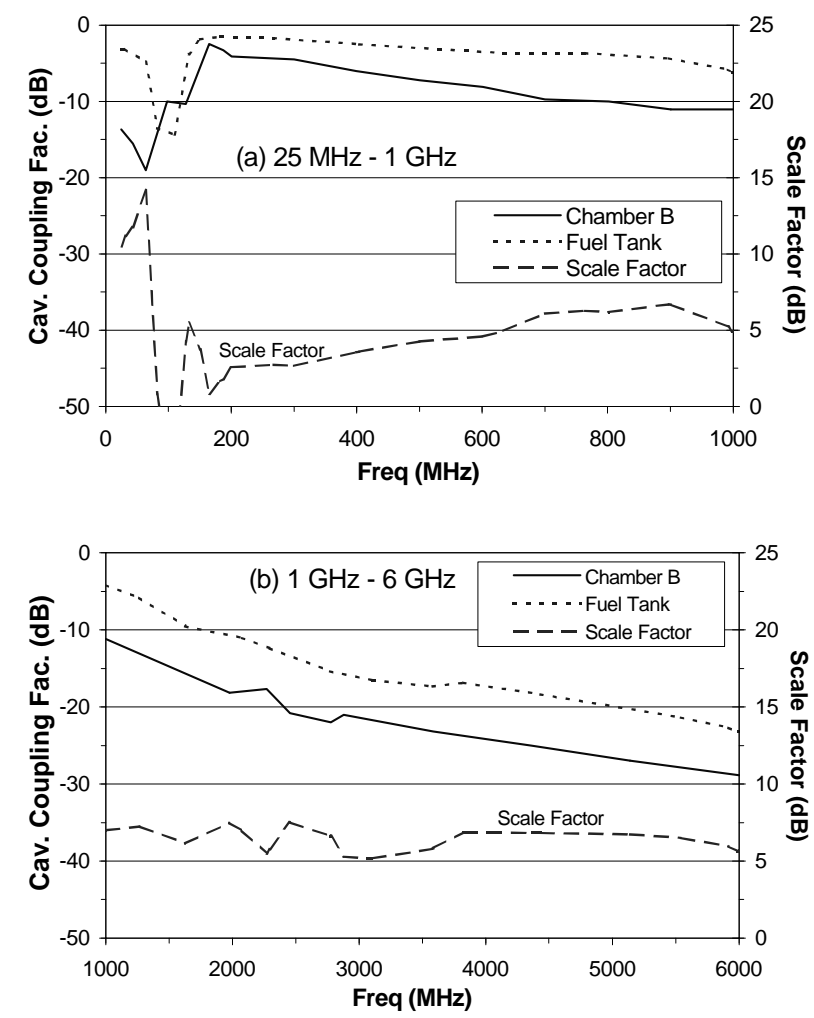

Figure 13. Cavity coupling envelopes and scale factor.

The same measurement procedure performed to characterize the CWT was repeated to characterize Reverberation Test Chamber "B". This chamber is a $2.9 m \times 3.96 m \times 7.01 m$ rectangular structure with painted steel walls. A large mechanical stirrer was positioned in a corner for stirring RF energy.

The approximate trend of the Chamber " $\mathrm{B}$ " results was plotted against the envelope of the CWT peak coupling factors in Figure 13. In addition, the scale factor, as computed from the ratio (or the difference in $\mathrm{dB}$ ) of the two data sets was also plotted on the second vertical axes. The scale factor represents the increase in input power to the reverberation chamber in order to achieve equivalent field strength as in the CWT.

\section{Bay 1 to Bay 3 Radiated Field Coupling}

A representative CWT bay 1 to bay 3 coupling measurement was made to provide an estimate of the typical shielding between any two bays. The coupling mechanism between any two bays can be radiated, such as through apertures and holes, or it can be conducted by wire coupling and then reradiate in other parts of the CWT.

Set up for this measurement is illustrated in Figure 14. In this setup, mode stirring was accomplished using two sets of stirrers, one in each bay. The rotational rates of the stirrers were selected to be different from one another, one at two seconds and the other at five seconds per revolution approximately, so as to maximize the number of stirrer position combinations. The spectrum analyzer sweep time was 20 seconds, and the trace was on maximum hold for about 2 minutes for each frequency. The measurements took approximately three hours for about 40 frequencies, including setup time. More details about the measurement method can be found in Reference [9].

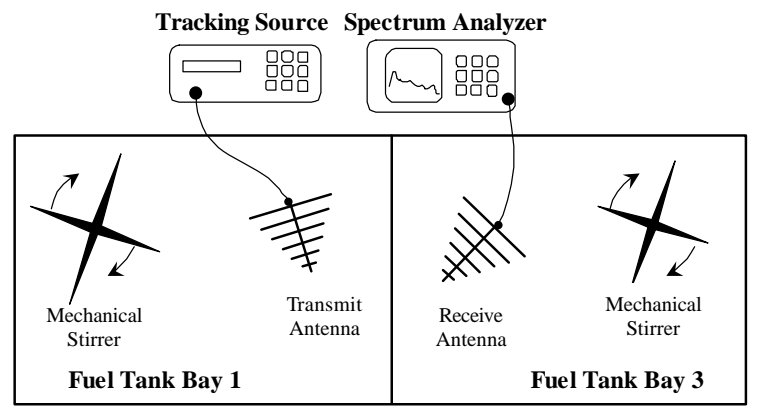

Figure 14. Bay 1 to bay 3 radiated field coupling.

The results are shown in Figure 15. In addition, the peak cavity coupling factors, where both transmit and receive antennas positioned in the same bay, are also shown for comparison.

An observation made from Figure 15 was that there was tight coupling (i.e. little shielding) between different bays below $1 \mathrm{GHz}$. In other words, the RF fields seen by wiring in other bays was nearly as high as that seen by the wires in the bay where the radiating source was located. This 
behavior justified the need to expose the whole wiring system when testing for susceptibility to RF radiated field, not just the wire section contained in one particular bay.

Above $1 \mathrm{GHz}$, the bay 1 to bay 3 coupling curve started to depart from the others. Above 3 $\mathrm{GHz}$, the separation was about $10 \mathrm{~dB}$, which was still not very large.

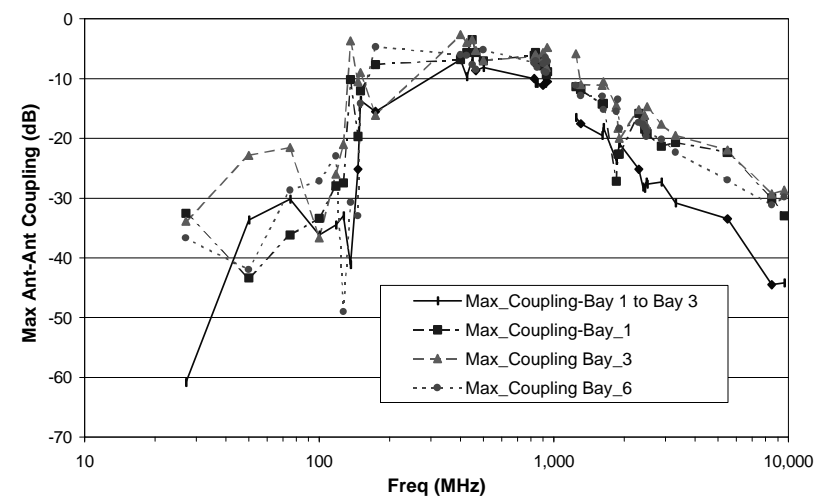

Figure 15. Bay 1 to bay 3 maximum radiated field coupling.

\section{RF Susceptibility Testing}

\section{Radiated Field Susceptibility}

NASA LaRC's Reverberation Test Chamber "B" was used to simulate the CWT radiated field threats. The test chamber input power levels were derived from the CWT radiated field threats, the CWT peak coupling factor envelope, and the CWTtest chamber scale factors. The required level of RF power was delivered into the test chamber through a log-periodic antenna. No sparking/arcing events were observed during the test. The system was not tested to failure, however.

Laboratory measurements at various points on the FQIS system indicated much higher voltage and current induced through conducted paths than through radiated fields [1], given the previously shown PEDs power level and measured coupling data. Thus, further testing was later performed with emphasis on susceptibility to conducted power.

\section{Conducted Power Susceptibility}

The power determined to exist on the wires from PEDs sources, as shown in Figure 5, was injected directly on the wires at the FQIS connector.
No arcing/sparking events were observed during the test. However, under simulated fault conditions (with the wires making intermittent shorts to ground), arcing/ sparking at the fault location was observed. More details on the testing are presented in References [1] and [2], with the test results included in Figure 16 of this paper.

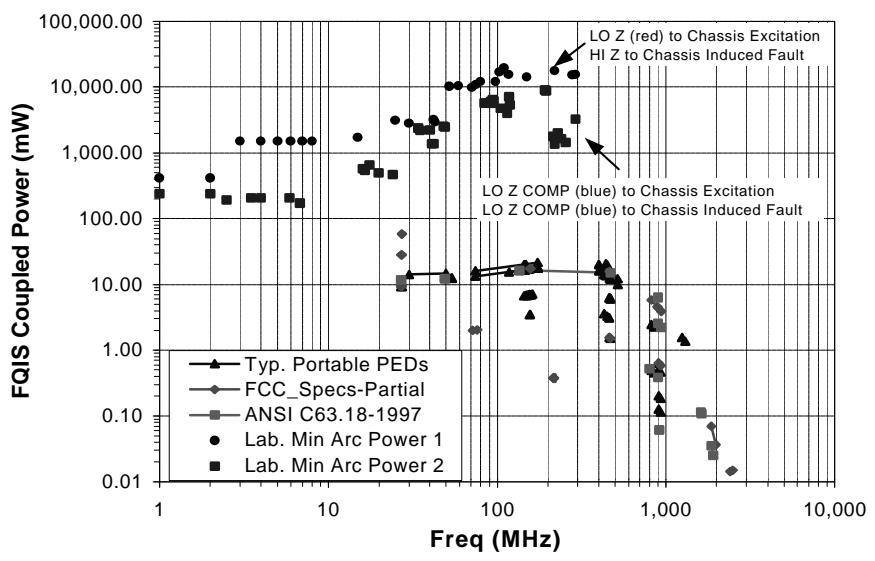

Figure 16. Adjusted PED threats on FQIS wiring. Also shown are FQIS minimum observed arc/spark levels with different excitation and induced faults (data from Reference [2]).

For the conducted susceptibility test, up to 40 $\mathrm{W}$ of power were injected onto the FQIS wires at the connector, while the simulated fault locations were monitored for arcing/sparking events. The artificial fault locations were selected to be at the wire terminals with the highest voltage/current relative to voltage/current at the connector.

The main question is whether the power needed to create a spark/arc exceeded the worst case power coupling onto the FQIS wiring. Figure 16 shows that the power needed to create an arc/spark, even with a fault condition, far exceeded the worst case coupling onto the FQIS wiring by a large margin. Even with a $25 \mathrm{~W}$ PED transmitter at $27 \mathrm{MHz}$, the safety margin was about $10 \mathrm{~dB}$. In the frequency range from $200 \mathrm{MHz}$ to $300 \mathrm{MHz}$, the safety margin was about $18 \mathrm{~dB}$. Above $300 \mathrm{MHz}$, a $40 \mathrm{~W}$ amplifier was not able to create a spark/arc.

\section{Conclusion}

This paper described the RF coupling measurements from portable electronic devices in the passenger cabin of a large transport aircraft to its fuel tank and wiring. The results were compared 
with laboratory simulations to determine the threats of creating arcing/sparking in the fuel tank.

The FQIS wiring was found to be the dominant coupling path for RF power into the fuel tank. The data obtained show that there was a minimum of 10 $\mathrm{dB}$ margin between the amount of power required to induce a spark/arc and the amount of power available from the PEDs, even with a $25 \mathrm{~W}$ source at $27 \mathrm{MHz}$. A spark/arc within the fuel tank would require an output power in excess of 250 watts from a single PED, or from multiple PEDs with frequencies and positions such that the effects are additive. This was assuming certain fault conditions existed on the wiring inside the fuel tank.

\section{Acknowledgement}

This work was supported by the National Transportation Safety Board and the Aviation Safety Program Office (AvSPO) at NASA Langley Research Center, Hampton, VA.

Contributions by Dr. R. E. Richardson were supported by the Joint Program Office for Special Technology Countermeasures (JPO-STC) of the Naval Surface Warfare Center, Dahlgren, VA.

\section{References}

[1] Ely, J. J., T. X. Nguyen, K. L. Dudley, S. A. Scearce, F. B. Beck, M. D. Desphande, and C. R. Cockrell, Mar. 2000, "Investigation of Electromagnetic Field Threat to Fuel Tank Wiring of a Transport Aircraft," NASA/TP-2000-209867.

[2] Ely, J. J., T. X. Nguyen, K. L. Dudley, S. A. Scearce, F. B. Beck, M. D. Deshpande, and C. R. Cockrell, Oct. 2000, "An Investigation of EME as a Potential Cause of Fuel Tank Ignition," Digital Avionics Systems Conference.

[3] Hughes, D. J., "Portable Electronic Devices (PEDs) Measured Emission Levels," P1625 E3 Support to the NTSB, 1999.

[4] ANSI C63.18-1997 "Recommended Practice for an On-Site, Ad Hoc Test Method for Estimating Radiated Electromagnetic Immunity of Medical Devices to Specific Radio-Frequency Transmitters."

[5] Ladbury, J., G. Koepke, D. Camell, Jan. 1998, "Evaluation of the NASA Langley Research Center
Mode-Stirred Chamber Facility," NIST Technical Note 1508.

[6] Crawford, M. L. and G.H. Koepke, Apr. 1986, "Design, Evaluation, and use of a Reverberation Chamber for Performing Electromagnetic Susceptibility/ Vulnerability Measurements," NBS Tech. Note 1092.

[7] Hill, D. A., J. W. Adams, M. T. Ma, A. R. Ondrejka, B. F. Riddle, M. L. Crawford and R. T. Johnk, Sept. 1993, "Aperture Excitation of Electrically Large, Lossy Cavities," NIST Tech. Note 1361.

[8] Johnson, D. M., M. O. Hatfield, M. B Slocum, T. A. Loughry, A. R. Ondrejka, R. T. Johnk, G. J. Freyer, Sept. 1997 , "Phase II Demonstration Test of The Electromagnetic Reverberation Characteristics of a Large Transport Aircraft," NSWCDD/TR-97/84.

[9] Hatfield, M. O., Aug. 1988, "Shielding Effectiveness Measurements using Mode-Stirred Chambers: A Comparison of Two Approaches," IEEE Trans. on Electromagnetic Compatibility, Vol. 30, No. 3. 